\title{
Osteogenic Effect of High Frequency Accelerations on Non-Weight Bearing Bones
}

\section{Mani Alikhani* and Cristina Teixeira}

Department of Orthodontics, New York University, New York, USA

The skeleton's ability to adapt to altered levels and patterns of mechanical loading can be used as a potential target for bone therapy. While extensive studies on the effect of exercise and external loading show an anabolic effect of mechanical stimulation on weight-bearing bones $[1,2]$, there are very few studies on how to use mechanical stimulation to increase bone formation in non-weight bearing bones such as alveolar bone (part of the upper and lower jaws that house the teeth). Resorption of this bone, which commonly occurs in response to periodontal disease or trauma, affects millions of people and can significantly decrease the long-term prognosis of the teeth that it supports. While many surgical and pharmaceutical methods to preserve alveolar bone have been suggested, they are all invasive, costly and have limited application. Therefore, the need to develop a safe mechanical regimen that can stimulate bone formation in alveolar bone is obvious. Unfortunately, due to different embryonic origins and mechanical environments, strategies that have been used to increase bone formation in weight-bearing bones cannot simply be adapted for non-weight bearing bones. Weight-bearing bones have endochondral origin, which enables growth under heavy mechanical loads-while the majority of craniofacial bones are not exposed to heavy loads, and form directly from mesenchymal cells (intramembranous origin) [3]. In addition, weight-bearing bones are exposed to direct loading, but alveolar processes are exposed to indirect loading via teeth and the periodontal ligament, which produces a complex pattern of strain distribution.

The main limiting factor in developing a mechanical regimen to increase bone formation in alveolar bone is our poor understanding of how mechanical stimulation can increase bone formation in the first place. Previously, it was assumed that the adaptation of bone depends on the magnitude of matrix deformation (strain). Therefore, it was presumed that to stimulate anabolic reaction in the bone a threshold of $0.1 \%$ strain would have to be exceeded [4]. Strains below this level of deformation were considered insufficient stimulation, and not effective in mitigating resorption [5]. In support of the assumption that matrix deformation is the critical parameter driving bone adaptation, many studies have focused on measuring the osteogenic effects of different properties of strain, such as strain magnitude [6], strain rate [7], electrokinetic streaming currents [8], piezoelectric currents [9], fluid shear flow [10] and strain energy density. In all these studies it was assumed that a high magnitude of matrix deformation is necessary for mechanical stimulation to be osteogenic. Unfortunately, mechanical intervention that relies on the application of large loads may be infeasible for many clinical situations that involve fragile bones. This is also obvious in the case of non-weight bearing bones. First, the majority of craniofacial bones are not exposed to heavy forces. Second, to be clinically practical these forces should be applied through the teeth with a minimum load to minimize tooth damage and discomfort. Third, in many clinical scenarios of alveolar bone loss or repair such as periodontal disease or early stages of dental implant integration, the application of high loads is contraindicated since they facilitate further bone loss. Therefore, to take advantage of the osteogenic effect of mechanical treatment in alveolar bone, other properties of mechanical stimulation must be considered.
While bone undeniably responds to high level of matrix deformation, it has been shown that increasing the frequency of the applied load stimulates bone formation in weight-bearing bones with a matrix deformation of less than $0.001 \%$ strain (11-12). The mechanism by which such low-level mechanical signals can cause bone formation is not clear. At this strain, the amount of matrix deformation is too small to be recognized by cells, therefore the idea that matrix deformation or byproducts of matrix deformation, such as streaming potentials, fluid drag on cellular processes, or enhanced nutrient transport [13-14], would be the sole factors responsible for bone formation is highly remote. These observations suggest that in addition to matrix deformation, mechanical stimulation may produce other signals that are not related to matrix deformation but are still osteogenic. In fact, it is more logical for cells to be sensitive to a direct stimulus that does not rely on matrix deformation, since this would reduce the complexity of the system and allow cells to respond to the mechanical environment without need for damage to the surrounding matrix. Recently, it has been suggested that physical acceleration of a cell may present such a signal [15].

While cells in different tissues such as bone, cartilage and ligament are exposed to different strains, they are all subject to similar accelerations, which increases the possibility that acceleration may serve as a more generic physical signal to control the adaptive response regardless of magnitude of the strain. Indeed, application of small oscillatory accelerations, independent of matrix deformation was able to enhance bone formation in weight-bearing bones [15]. This property of mechanical stimulation can become very useful in the development of a mechanical regimen to increase bone formation in non-weight bearing bones, since it does not rely on application of high loads.

We recently were able to significantly increase bone formation in alveolar bone by applying high frequency $(60 \mathrm{~Hz})$ acceleration $(0.3 \mathrm{~g})$ with almost absent matrix deformation [16]. In our study, the osteogenic effect of high frequency accelerations (HFA) exhibited a gradient response, suggesting an anabolic effect on adjacent alveolar bone that is distant from the point of application. This is clinically very significant, because this procedure permits even safer increases in bone formation in fragile areas by application of the stimuli on teeth away from those areas.

It should be emphasized that higher accelerations are usually

*Corresponding author: Mani Alikhani, Department of Orthodontics, New York University, New York, USA, Tel: 212-998-9224; E-mail: ma343@nyu.edu

Received September 27, 2012; Accepted September 28, 2012; Published October 01, 2012

Citation: Alikhani M, Teixeira C (2012) Osteogenic Effect of High Frequency Accelerations on Non-Weight Bearing Bones. J Tissue Sci Eng 3:e115. doi:10.4172/2157-7552.1000e115

Copyright: (c) 2012 Alikhani M, et al. This is an open-access article distributed under the terms of the Creative Commons Attribution License, which permits unrestricted use, distribution, and reproduction in any medium, provided the original author and source are credited. 
Citation: Alikhani M, Teixeira C (2012) Osteogenic Effect of High Frequency Accelerations on Non-Weight Bearing Bones. J Tissue Sci Eng 3:e115. doi:10.4172/2157-7552.1000e115

accompanied by higher strains, which limits the application of higher acceleration as the sole osteogenic source in the mouth. Changes in mechanical stimulation frequency are a safe compensation for this shortcoming. While the mechanism through which acceleration can stimulate bone formation is not clear, it has been suggested that oscillation of nuclei in cytoplasm may activate cytoskeleton, which may increase osteoblast activity. Indeed, in our experiment we found significant increases in osteoblast activity (versus proliferation), as demonstrated by the increase in expression of genes that participate in different stages of bone formation, from matrix synthesis to mineralization.

Other therapeutic modalities, such as ultrasound [17], electric fields [9], and magnetic fields [18] have been suggested to increase bone formation. Unfortunately, the cost and complexity of these approaches have limited their application to alveolar bone. Our studies suggest a simple mechanical recipe that may play a significant role in alveolar bone formation and maintenance. Further research in this field would help us understand the mechanism of adaptation developed by our skeleton in response to mechanical stimulation during evolution, and the application of this mechanism for treatment of bone loss and injuries in non-weight bearing bones.

\section{References}

1. Honda A, Umemura Y, Nagasawa S (2001) Effect of high-impact and lowrepetition training on bones in ovariectomized rats. J Bone Miner Res 16: 16881693.

2. Tanaka SM, Alam IM, Turner CH (2003) Stochastic resonance in osteogenic response to mechanical loading. Faseb j 17: 313-314.

3. Teixeira CC, Liu Y, Thant LM, Pang J, Palmer G, et al. (2010) Foxo1, a novel regulator of osteoblast differentiation and skeletogenesis. J Biol Chem 285: 31055-31065.

4. Turner CH, Forwood MR, Rho JY, Yoshikawa T (1994) Mechanical loading thresholds for lamellar and woven bone formation. J Bone Miner Res 9: 87-97.
5. Frost HM (1987) Bone "mass" and the "mechanostat": a proposal. Anat Rec 219: $1-9$.

6. Rubin CT, Lanyon LE (1985) Regulation of bone mass by mechanical strain magnitude. Calcif Tissue Int 37: 411-417.

7. O'Connor JA, Lanyon LE, MacFie H (1982) The influence of strain rate on adaptive bone remodelling. J Biomech 15: 767-781.

8. Pollack SR, Salzstein R, Pienkowski D (1984) The electric double layer in bone and its influence on stress-generated potentials. Calcif Tissue Int 36: S77-S81.

9. Bassett ca, pawluk rj, becker ro (1964) effects of electric currents on bone in vivo. Nature 204: 652-654.

10. Weinbaum S, Cowin SC, Zeng Y (1994) A model for the excitation of osteocytes by mechanical loading-induced bone fluid shear stresses. J Biomech 27: 339 360

11. Oxlund BS, Ørtoft G, Andreassen TT, Oxlund H (2003) Low-intensity, highfrequency vibration appears to prevent the decrease in strength of the femu and tibia associated with ovariectomy of adult rats. Bone 32: 69-77.

12. Rubin C, Turner AS, Bain S, Mallinckrodt C, McLeod K (2001) Anabolism. Low mechanical signals strengthen long bones. Nature 412: 603-604.

13. Qin YX, Kaplan T, Saldanha A, Rubin C (2003) Fluid pressure gradients, arising from oscillations in intramedullary pressure, is correlated with the formation of bone and inhibition of intracortical porosity. J Biomech 36: 1427-1437.

14. Malone AM, Batra NN, Shivaram G, Kwon RY, You L, et al. (2007) The role of actin cytoskeleton in oscillatory fluid flow-induced signaling in MC3T3-E1 osteoblasts. Am J Physiol Cell Physiol 292: C1830-1836.

15. Garman R, Gaudette G, Donahue LR, Rubin C, Judex S (2007) Low-leve accelerations applied in the absence of weight bearing can enhance trabecular bone formation. J Orthop Res 25: 732-740.

16. Alikhani M, Khoo E, Alyami B, Raptis M, Salqueiro JM, et al. (2012) Osteogenic effect of high-frequency acceleration on alveolar bone. J Dent Res 91: 413-419.

17. Yang KH, Parvizi J, Wang SJ, Lewallen DG, Kinnick RR, et al. (1996) Exposure to low-intensity ultrasound increases aggrecan gene expression in a rat femur fracture model. J Orthop Res 14: 802-809.

18. Xu S, Tomita N, Ohata R, Yan Q, Ikada Y (2001) Static magnetic field effects on bone formation of rats with an ischemic bone model. Biomed Mater Eng 11 257-263. 